\section{Effect of Aminoethoxyvinylglycine (AVG) on Preharvest Drop, Fruit Quality, and Maturation of 'McIntosh' Apples. II. Effect of Timing and Concentration Relationships and Spray Volume}

\author{
Duane W. Greene \\ Department of Plant and Soil Sciences, University of Massachusetts, Amherst, \\ MA 01003
}

\author{
James R. Schupp \\ Cornell University, Hudson Valley Laboratory, Highland, NY 12528
}

Additional index words. ReTain, ethylene, ripening, abscission, harvest management, daminozide, NAA, plant growth regulator

\begin{abstract}
AVG was evaluated for its effect on controlling preharvest drop and influencing ripening of 'McIntosh' apples in Maine and Massachusetts. AVG consistently and effectively retarded preharvest drop. AVG was superior to NAA and comparable to daminozide in drop control. Dilute or $2 \times$ applications were more effective than applications made at lower water volumes. One application of AVG made 4 weeks before anticipated normal harvest was more effective in controlling preharvest drop than split applications of the same amount made earlier or later. In general, AVG delayed ripening as assessed by a retardation in the development of red color, maintenance of flesh firmness, delayed degradation of starch, and a delayed onset of the ethylene climacteric. We conclude that AVG is an effective drop control compound that is also useful as a management tool to extend the harvest window for blocks of 'McIntosh' that would otherwise ripen simultaneously. Chemical names used: aminoethoxyvinylglycine (AVG), naphthaleneacetic acid (NAA), succinic acid-2,2-dimethylhydrazide (daminozide, Alar).
\end{abstract}

For a number of years growers in the Northeast had largely depended upon daminozide to control preharvest drop, retard ripening, and allow an orderly harvest of the 'McIntosh' apple crop. Little effort was put forth to reevaluate old drop control materials, or look for new chemical solutions because daminozide proved to be such a satisfactory compound. The withdrawal of the registration of daminozide for use on apples stimulated new interest in looking for alternative ways to accomplish these goals and to allow an orderly harvest of 'McIntosh' apples (Shafer et al., 1995). One such material was AVG. A review of early research on AVG is contained in the first paper in this series (Schupp and Greene, 2003). Early work focused on evaluating handgun applications of relatively high concentrations of AVG, applied to the drip point, a method that does not reflect commercial spray application technology. In order to make recommendations to apple growers about the application of AVG it was necessary to evaluate efficacy of AVG applied by air-blast sprayers at commonly used spray volumes. Multiple applications of plant growth regulators may improve efficacy. Marini et al. (1993) showed that repeated applications of naphthaleneacetic acid (NAA) delayed fruit abscission more that a single application.

This paper examines the effect of time, concentration, and spray volume of AVG on

Received for publication 27 Jan. 2003. Accepted for publication 6 Oct. 2003. water, $60 \%$ of volume required for a dilute application based upon calculated tree row volume (TRV). The effect of water volume was assessed by applying $351 \mathrm{~g} \cdot \mathrm{ha}^{-1} \mathrm{AVG}$ in $0.1 \%$ ABG-7034 surfactant in 189,379 , or $568 \mathrm{~L}$ applied by airblast sprayer or in $946 \mathrm{~L}$ of water applied by a handgun. Treatments were applied on 25 and 26 Aug., 1992. Naphthaleneacetic acid (NAA, Fruitone N, AMVAC Corp., Newport Beach, Calif.)was applied at $20 \mathrm{mg} \cdot \mathrm{L}^{-1}$ on $16 \mathrm{Sept}$.

Fruit quality at harvest was assessed at four weekly intervals starting on 21 Sept. Twenty fruit were sampled randomly from the periphery of the tree at each sampling date. Fruit diameter and weight were determined and then the percent red color on each fruit was estimated to the nearest $10 \%$. Flesh firmness was determined by puncturing each fruit once with a press- mounted Effegi penetrometer (McCormick Fruit Co., Yakima, Wash.). A composite sample of juice collected during the pressure test was used to measure soluble solids with a hand-held refractometer (Mc Cormick Fruit Co.). Starch status of the fruit was rated using the method of Priest and Lougheed (1981). The chroma and hue of the red blush of each fruit was measured with a chromameter (Minolta, Corp., Ramsen, N.J.).

Ten fruit per tree were randomly sampled at weekly intervals from several treatments for determination of internal ethylene using the method of Bramlage et al. (1980). A 1-ml air sample from the core cavity of each fruit was injected into a gas chromatograph equipped with an alumina column and an FID detector. The resulting peaks were compared to that of a $10 \mu \mathrm{L} \cdot \mathrm{L}^{-1}$ ethylene standard and the internal ethylene concentration (IEC) was calculated.

Drop was assessed by tagging one or two limbs per tree that contained 40 fruit. The fruit were counted three times per week for four weeks. Fruit drop for each two or three-d interval was calculated by subtracting the current count from the initial count.

On the second harvest date, 28 Sept., an additional fruit sample was collected and placed in a nonsegregated air storage held at 1 to $3{ }^{\circ} \mathrm{C}$. Fruit were removed after $150 \mathrm{~d}$ and weight, flesh firmness, decay and physiological disorders evaluated.

Experiment 2. Concentration, Massachusetts. AVG was applied to 'Marshall McIntosh' as the ABG-3097 formulation in 0.1\% ABG7034 surfactant at rates of $62,124,247,371$, 494, and $618 \mathrm{~g} \cdot \mathrm{ha}^{-1}$ a.i. in $936 \mathrm{~L} \cdot \mathrm{ha}^{-1}$ of water (80\% tree row volume dilute) on 24 Aug. 1992. NAA was applied at $10 \mathrm{mg} \cdot \mathrm{L}^{-1}$ on 8 Sept. Fruit quality was assessed at harvest by taking four weekly samples starting on 15 Sept. On each harvest date a 20-fruit sample was taken at random around the periphery of the tree. It was weighed and then red color was estimated on each fruit to the nearest $10 \%$. Ten apples characteristic of the sample were selected and flesh firmness determined on two sides with an Effegi penetrometer. Soluble solids and starch rating were done as previously described.

All fruit were picked up under the trees designated for evaluation of drop on 31 Aug. Starting the first week in September and extending through 9 Nov., fruit was picked up under these 
trees twice per week and counted. At that time all remaining fruit on the tree were harvested and counted. Drop was calculated based upon the total number of fruit on the tree.

Experiment 3: Watervolume, Massachusetts. AVG was applied to 'Marshall McIntosh' as the ABG-3097 formulation in $0.1 \%$ ABG7034 surfactant a rate of $175 \mathrm{~g} \cdot \mathrm{ha}^{-1}$ a.i. in 189 , 284,379 , or $473 \mathrm{~L} \cdot \mathrm{ha}^{-1}$ of water (full dilute tree row volume) on 25 Aug., 1992. NAA was applied at $10 \mathrm{mg} \cdot \mathrm{L}^{-1}$ on $8 \mathrm{Sept}$. Fruit quality was assessed at harvest by taking four weekly samples starting on 17 Sept. Fruit quality and preharvest drop were assessed similar to that described for Expt. 2.

Experiment 4. Concentration and multiple applications, Maine. Two formulations of AVG were evaluated; an $85 \%$ formulation, ABG3097, used previously and ABG-3168 (Abbott laboratories, North Chicago, Ill.), a 15\% technical powder. Both formulations were applied at $124 \mathrm{gha}^{-1}$ a.i. at 6,4 , and 2 weeks before harvest (WBH) on 9Aug., 23 Aug. and 6Sept. The AVG formulations were also applied at $185 \mathrm{~g} \cdot \mathrm{ha}^{-1}$ a.i. on 23 Aug. and 6 Sept., and $371 \mathrm{~g} \cdot \mathrm{ha}^{-1}$ a.i. was applied on $23 \mathrm{Aug}$., $4 \mathrm{WBH}$. One group of trees received AVG as the ABG-3168 formulation on 23 Aug., and one group was unsprayed and served as the control. All AVG treatments were applied in $1235 \mathrm{~L} \cdot \mathrm{ha}^{-1}$ water (TRV 2338 $\mathrm{L} \cdot \mathrm{ha}^{-1}$ ) and ABG-7034 surfactant at $0.1 \%(\mathrm{v} / \mathrm{v})$ was included. NAA at $10 \mathrm{mg} \cdot \mathrm{L}^{-1}$ was applied to a final group of trees on 6 and 13 Sept. Fruit quality and preharvest drop, were determined in a similar manner to that described in Expt. 1. Ten fruit were sampled each week, starting 20 Sept., for IEC analysis. The evaluation was similar to that described in Expt. 1.

Experiment 5. Concentration and multiple applications, Massachusetts. AVG as the ABG3168 formulation was applied at 124, 185 and $371 \mathrm{~g} \cdot \mathrm{ha}^{-1}$ a.i. at 6,4 , and $2 \mathrm{WBH}$ on 5 Aug., 19 Aug., and 2 Sept. as described in Expt. 4. One group of trees received $750 \mathrm{mg} \cdot \mathrm{L}^{-1}$ daminozide on 5 Aug. and another $10 \mathrm{mg} \cdot \mathrm{L}^{-1} \mathrm{NAA}$ on 9 Sept. All treatments were applied in 1122
$\mathrm{L} \cdot \mathrm{ha}^{-1}$ of water with $0.1 \%$ (v/v) ABG-7034 surfactant. Twenty fruit per tree were harvested at four weekly intervals starting on $16 \mathrm{Sept}$. and fruit quality assessed as described in Expt. 3. All fruit were picked up under trees on 3 Sept. and drop determined until 8 Nov. as described in Expt. 3.

Analysis of variance was used in all experiments to determine significance of treatments. Where appropriate, means were separated by orthogonal polynomial comparison, analysis of regression, or Duncan's multiple range test.

\section{Results}

Experiment 1. Concentration and water volume, Maine. AVG application delayed preharvest drop linearly with increasing concentration (Table 1). 'McIntosh' is normally harvested by the end of September, and $10 \%$ preharvest drop is an arbitrary threshold for acceptable drop control. It appears that at least $371 \mathrm{~g} \cdot \mathrm{ha}^{-1} \mathrm{a}$.i. was required to achieve this level of drop control.

Table 1. Effect of AVG concentration and spray volume, and NAA on preharvest drop of 'McIntosh' apples, Maine, 1992, Expt. 1.

\begin{tabular}{|c|c|c|c|c|c|c|c|c|c|c|c|c|c|}
\hline \multicolumn{2}{|c|}{$\mathrm{AVG}^{\mathrm{z}}$} & \multirow{2}{*}{$\begin{array}{c}\text { Spray } \\
\text { vol } \\
\left(\mathrm{L} \cdot \mathrm{ha}^{-1}\right)\end{array}$} & \multicolumn{11}{|c|}{ Cumulative drop (\%) } \\
\hline (g.ha-1 a.i.) & (g/acre a.i.) & & 23 Sept. & 25 Sept. & 28 Sept. & 30 Sept. & 2 Oct. & 5 Oct. & 7 Oct. & 9 Oct. & 12 Oct. & 14 Oct. & 16 Oct. \\
\hline Control 0 & $(0)$ & --- & 3 & 3 & 8 & 14 & 15 & 22 & 27 & 46 & 62 & 76 & 87 \\
\hline 124 & (50) & 568 & 2 & 3 & 4 & 11 & 13 & 19 & 29 & 44 & 66 & 75 & 85 \\
\hline 248 & $(100)$ & 568 & 1 & 2 & 7 & 8 & 11 & 16 & 19 & 28 & 52 & 68 & 83 \\
\hline 371 & $(150)$ & 568 & 2 & 3 & 3 & 4 & 4 & 4 & 6 & 17 & 39 & 46 & 57 \\
\hline 494 & (200) & 568 & 2 & 2 & 4 & 5 & 6 & 7 & 8 & 13 & 36 & 38 & 45 \\
\hline 618 & $(250)$ & 568 & 0 & 0 & 3 & 4 & 5 & 5 & 5 & 9 & 18 & 24 & 35 \\
\hline 741 & $(300)$ & 568 & 0 & 0 & 0 & 1 & 2 & 2 & 2 & 3 & 6 & 15 & 16 \\
\hline 351 & & $189(5 \times)$ & 1 & 1 & 3 & 4 & 5 & 14 & 15 & 22 & 48 & 58 & 68 \\
\hline 351 & & $379(3 \times)$ & 1 & 1 & 2 & 2 & 3 & 4 & 5 & 13 & 32 & 44 & 61 \\
\hline 351 & & $568(2 \times)$ & 0 & 0 & 1 & 1 & 1 & 2 & 3 & 12 & 18 & 28 & 44 \\
\hline 351 & & 946 (dilute) & 3 & 3 & 3 & 4 & 5 & 5 & 5 & 6 & 16 & 24 & 33 \\
\hline NAA $20 \mathrm{mg}$ & & 568 & 3 & 3 & 5 & 9 & 10 & 21 & 28 & 46 & 73 & 77 & 79 \\
\hline \multicolumn{14}{|c|}{ Significance } \\
\hline AVG co & ntration & & NS & $\mathrm{L}^{*}$ & $\mathrm{~L}^{*}$ & $\mathrm{~L}^{* * *}$ & $\mathrm{~L}^{* * * *}$ & $\mathrm{~L}^{* * *}$ & $\mathrm{~L}^{* * *}$ & $\mathrm{~L}^{* * *}$ & $\mathrm{~L}^{* * *}$ & $\mathrm{~L}^{* * *}$ & $\mathrm{~L}^{* * *}$ \\
\hline Water v & & & NS & NS & NS & NS & $\mathrm{L}^{* *}$ & NS & NS & NS & $\mathrm{L}^{* * * *}$ & $\mathrm{~L}^{* * *}$ & $\mathrm{~L}^{* * * *}$ \\
\hline NAA vs & ntrol & & NS & NS & $*$ & $*$ & $*$ & NS & NS & NS & $*$ & NS & NS \\
\hline NAA vs & & & NS & NS & NS & NS & NS & $*$ & $*$ & $* *$ & $* * *$ & $* * *$ & $* *$ \\
\hline
\end{tabular}

${ }^{7}$ AVG was applied 25 and 26 Aug. 1992 and naphthaleneacetic acid was applied on 16 Sept. 1992.

ss,*,******Nonsignificant or significant at $P=0.05,0.01$, or 0.001 , respectively; $\mathrm{L}=$ linear response.

Table 2. Effect of aminoethoxyvinylglycine (AVG) concentration and spray volume on fruit characteristics at harvest, Maine, 1992 , Expt. 1.

\begin{tabular}{|c|c|c|c|c|c|c|c|c|c|}
\hline $\begin{array}{l}\mathrm{AVG}^{\mathrm{z}} \\
\left(\mathrm{g} \cdot \mathrm{ha}^{-1} \text { a.i. }\right)\end{array}$ & $\begin{array}{l}\text { Spray } \\
\text { vol } \\
\left(\mathrm{L} \cdot \mathrm{ha}^{-1}\right)\end{array}$ & $\begin{array}{c}\text { Fruit } \\
\text { wt } \\
(\mathrm{g})\end{array}$ & $\begin{array}{c}\text { Red } \\
\text { color } \\
(\%)\end{array}$ & $\begin{array}{l}\text { Fruit } \\
\text { diam } \\
(\mathrm{mm})\end{array}$ & $\begin{array}{c}\text { Color } \\
\text { L* }\end{array}$ & $\begin{array}{l}\text { Hue } \\
\text { angle }\end{array}$ & $\begin{array}{c}\text { Flesh } \\
\text { firmness } \\
(\mathrm{N})\end{array}$ & $\begin{array}{c}\text { Soluble } \\
\text { solids } \\
(\%)\end{array}$ & $\begin{array}{l}\text { Starch } \\
\text { rating } \\
(1-9)\end{array}$ \\
\hline \multicolumn{10}{|c|}{ Mean of harvest on 21 and 28 Sept., 5 and 12 Oct. } \\
\hline AVG 0 (control) & --- & 156 & 70 & 74 & 35.0 & 34.6 & 55.1 & 10.5 & 6.7 \\
\hline AVG 124 & 568 & 163 & 64 & 76 & 35.5 & 35.8 & 54.9 & 10.6 & 6.6 \\
\hline AVG 248 & 568 & 159 & 66 & 75 & 35.1 & 34.6 & 5.5 & 10.3 & 6.2 \\
\hline AVG 371 & 568 & 166 & 67 & 76 & 34.6 & 33.0 & 57.1 & 10.9 & 5.8 \\
\hline AVG 494 & 568 & 167 & 69 & 76 & 34.8 & 32.9 & 56.3 & 11.0 & 5.9 \\
\hline AVG 618 & 568 & 172 & 68 & 77 & 34.7 & 32.6 & 57.8 & 10.9 & 5.3 \\
\hline AVG 741 & 568 & 168 & 60 & 76 & 35.4 & 35.2 & 57.4 & 10.9 & 5.7 \\
\hline AVG 351 & $189(5 \times)$ & 163 & 69 & 75 & 35.8 & 34.5 & 58.0 & 10.6 & 6.1 \\
\hline AVG 351 & $379(3 \times)$ & 161 & 71 & 75 & 34.7 & 33.0 & 57.7 & 10.8 & 5.9 \\
\hline AVG 351 & $568(2 \times)$ & 168 & 64 & 76 & 35.3 & 34.6 & 57.2 & 10.9 & 5.6 \\
\hline AVG 351 & 946 (dilute) & 165 & 63 & 75 & 35.1 & 34.1 & 57.3 & 10.7 & 5.8 \\
\hline $\mathrm{NAA} 20 \mathrm{mg} \cdot \mathrm{L}^{-1}$ & 568 & 173 & 71 & 77 & 34.6 & 35.3 & 52.4 & 10.9 & 7.0 \\
\hline \multicolumn{10}{|l|}{ Significance } \\
\hline AVG concentration & & $\mathrm{L}^{* *}$ & NS & $\mathrm{L}^{*}$ & NS & NS & $\mathrm{L}^{* *}$ & $\mathrm{~L}^{* *}$ & $\mathrm{~L}^{* * * *}$ \\
\hline Harvest time & & $* * *$ & $* * *$ & $* * *$ & $* * *$ & $* * *$ & $* * *$ & $* * *$ & $* * *$ \\
\hline AVG $\times$ time & & NS & NS & NS & NS & NS & $* * *$ & NS & $* *$ \\
\hline Spray volume & & NS & NS & NS & NS & NS & NS & NS & NS \\
\hline NAA vs. control & & NS & NS & NS & NS & NS & $*$ & NS & NS \\
\hline NAA vs. AVG & & NS & NS & NS & NS & NS & $* * *$ & NS & $* * *$ \\
\hline
\end{tabular}

${ }^{2}$ AVG was applied on 25 and 26 Aug. 1992 and naphthaleneacetic acid was applied on 16 Sept. 1992.

Ns, ,**,*****Nonsignificant or significant at $P=0.05,0.01$, or 0.001 , respectively; $\mathrm{L}=$ linear response. 
Table 3. Effect of aminoethoxyvinylglycine (AVG) concentration and spray volume on internal ethylene concentration, Maine, 1992, Expt. 1.

\begin{tabular}{|c|c|c|c|c|c|c|}
\hline \multirow{2}{*}{$\begin{array}{l}\mathrm{AVG}^{\mathrm{z}} \\
\left(\mathrm{g} \cdot \mathrm{ha}^{-1} \text { a.i. }\right)\end{array}$} & \multirow{2}{*}{$\begin{array}{c}\text { Spray } \\
\text { vol } \\
\left(\mathrm{L} \cdot \mathrm{ha}^{-1}\right)\end{array}$} & \multicolumn{5}{|c|}{ Internal ethylene concn $\left(\mathrm{mg} \cdot \mathrm{L}^{-1}\right)$} \\
\hline & & $\overline{\mathrm{H} 1-\mathrm{H} 4^{\mathrm{y}} \text { (mean) }}$ & 21 Sept. & 28 Sept. & 5 Oct. & 12 Oct. \\
\hline AVG 0 (control) & 568 & 14.6 & 3.2 & 10.1 & 19.3 & 32.4 \\
\hline AVG 124 & 568 & 13.2 & 1.0 & 8.1 & 21.8 & 31.2 \\
\hline AVG 248 & 568 & 9.3 & 0.9 & 2.2 & 7.2 & 32.1 \\
\hline AVG 741 & 568 & 3.7 & 0 & 0.1 & 0.3 & 19.0 \\
\hline AVG 351 & $189(5 \times)$ & 7.9 & 0.1 & 0.3 & 5.0 & 29.8 \\
\hline AVG 351 & $568(1.5)$ & 5.5 & 2.4 & 0 & 1.2 & 25.4 \\
\hline AVG 351 & $946(1 \times)$ & 4.5 & 0 & 0.7 & 2.6 & 21.0 \\
\hline \multicolumn{7}{|l|}{ Significance } \\
\hline AVG concentration & & $\mathrm{L}^{* * *}$ & NS & $\mathrm{L}^{* * *}$ & $\mathrm{~L}^{* * *}$ & $\mathrm{~L}^{* * *}$ \\
\hline Harvest time & & $* * *$ & & & & \\
\hline AVG volume & & NS & NS & NS & $\mathrm{L}^{*}$ & $\mathrm{~L}^{*}$ \\
\hline AVG $\times$ time & & $* * *$ & & & & \\
\hline AVG vs. control & & $* * *$ & NS & $* * *$ & * & NS \\
\hline
\end{tabular}

${ }^{2}$ AVG was applied 25 and 26 Aug. 1992 and naphthaleneacetic acid on 16 Sept. 1992.

${ }^{y}$ Mean internal ethylene concentration across all four harvest dates.

ss,*,*** Nonsignificant or significant at $P=0.05$ or 0.001 , respectively; $\mathrm{L}=$ linear response.

Table 4. Effect of aminoethoxyvinylglycine (AVG) concentration and naphthaleneacetic acid (NAA) on cumulative drop of 'Marshall McIntosh'/M.26 apples. Massachusetts, 1992.

\begin{tabular}{|c|c|c|c|c|c|c|c|}
\hline \multirow{2}{*}{$\begin{array}{l}\text { Treatment }^{2} \\
\left(\mathrm{~g} \cdot \mathrm{ha}^{-1} \text { a.i. }\right)\end{array}$} & \multicolumn{7}{|c|}{ Cumulative drop (\% of total on tree) } \\
\hline & Sept. $7^{y}$ & Sept. 14 & Sept. 21 & Sept. 28 & Oct. 5 & Oct. 12 & Oct. 19 \\
\hline Control 0 & 1 & 2 & 16 & 35 & 64 & 89 & 97 \\
\hline AVG 61.8 & 1 & 3 & 9 & 23 & 57 & 90 & 97 \\
\hline AVG 123.5 & 1 & 1 & 8 & 22 & 51 & 83 & 94 \\
\hline AVG 247.0 & 1 & 2 & 6 & 12 & 25 & 61 & 81 \\
\hline AVG 371.5 & 1 & 1 & 3 & 8 & 26 & 55 & 75 \\
\hline AVG 494.0 & 1 & 1 & 2 & 5 & 12 & 43 & 67 \\
\hline AVG 617.5 & 1 & 1 & 2 & 3 & 6 & 18 & 356 \\
\hline NAA $10 \mathrm{mg} \cdot \mathrm{L}^{-1}$ & 1 & 2 & 16 & 41 & 69 & 90 & 97 \\
\hline \multicolumn{8}{|l|}{ Significance } \\
\hline AVG & $\mathrm{L}^{*}$ & $\mathrm{~L}^{*}$ & $\mathrm{~L}^{* * *} \mathrm{Q}^{*}$ & $\mathrm{~L}^{* * * *}$ & $\mathrm{~L}^{* * * *}$ & $\mathrm{~L}^{* * *} \mathrm{Q}^{*}$ & $\mathrm{~L}^{\mathrm{***}} \mathrm{Q}^{* * * *}$ \\
\hline AVG vs. NAA & NS & $* * *$ & $* * *$ & $* * *$ & $* * *$ & $* * *$ & $* * *$ \\
\hline Control vs. NAA & NS & NS & NS & NS & NS & NS & NS \\
\hline
\end{tabular}

${ }^{2}$ AVG was applied 24 Aug. 1992 and naphthaleneacetic acid on 9 Sept. 1992.

${ }_{\text {Ns, }, *, * * * * *}$ Nonsignificant or significant at $P=0.05,0.01$, or 0.001 , respectively; $\mathrm{L}=$ linear and $\mathrm{Q}=$ quadratic response.

Drop control was best when AVG was applied as a full dilute rate $(1 \times)$ or in the $2 x$ volume. As the amount of water that AVG was applied to the tree in was reduced there was a linear reduction in the drop, first becoming apparent on 2 Oct. and becoming highly significant on 12 Oct. and later. NAA reduced drop marginally for a little more than 1 week. During the majority of the season, AVG was superior to NAA as a drop control agent.

AVG significantly and linearly increased fruit weight, fruit diameter, and soluble solids
(Table 2). AVG maintained flesh firmness and delayed ripening based on starch index, but there was an AVG $\times$ time interaction for both factors. AVG reduced flesh firmness only on the last harvest date, 12 Oct. (data not shown). Conversely, AVG delayed the loss of starch from the fruit for the first three harvests, but not on the final harvest date. The volume of water used to deliver AVG did not influence any fruit quality parameter. NAA advanced maturity relative to AVG based upon color, flesh firmness, and starch rating.
IEC was reduced linearly by AVG but there was an AVG $\times$ time interaction (Table 3). IEC was reduced uniformly by all AVG concentrations during the first harvest but at the later harvests there was a linear reduction with increasing concentration. Overall, spray volume did not influence ethylene evolution. However, while spray volume did not influence fruit ethylene during the first harvest, a significant inverse relationship between spray volume and ethylene evolution was detected for the last two harvests.

AVG increased the amount of superficial scald that developed on fruit during $150 \mathrm{~d}$ regular air storage (storage data not shown). Fruit wight was greater for AVG-treated fruit following storage. AVG spray volume did not influence any variables measured. NAA reduced flesh firmness of fruit following storage.

Experiment 2. Concentration, Massachusetts. AVG retarded preharvest drop over an 8 -week period. The response was primarily linear early in the season when fruit would have been harvested commercially (Table 4). Normal commercial harvest in this block would have occurred between 20 Sept. and 1 Oct. Therefore, it appears that at least $247 \mathrm{~g} \cdot \mathrm{ha}^{-1} \mathrm{a}$.i. was required to get a good commercial control of drop $(10 \%$ or less) to the end of the harvest season. AVG was superior to NAA as a drop control agent except on the first evaluation date when there was less than $2 \%$ drop. AVG maintained flesh firmness and reduced fruit red color development, while NAA reduced red color but increased starch rating (data not shown).

Experiment 3. Water volume, Massachusetts. AVG retarded drop until from 21 Sept. to 19 Oct.. (Table 5). AVG applied in a larger volume of water appeared to more effectively retard preharvest drop, although to only a small extent. At no time during the drop collection period did NAA controlled drop relative to the control. AVG retarded red color development and spray volume appeared to have little influence on fruit quality (data not shown).

Experiment 4. Concentration and multiple applications, Maine. Both formulations of AVG, ABG-3097 and ABG-3168, reduced preharvest drop (Table 6), but the technical $15 \%$ formulation, ABG-3168 was more effective on the last two drop evaluation days. Drop control from the $372 \mathrm{~g} \cdot \mathrm{ha}^{-1}$ a.i. rate through the first three

Table 5. Effect of volume of water aminoethoxyvinylglycine (AVG) (175 g.ha-1 a.i.) was applied in and napthaleneacetic acid (NAA) on fruit cumulative drop on 'Marshall McIntosh'/M.26 apples, Massachusetts, 1992, Expt. 3.

\begin{tabular}{|c|c|c|c|c|c|c|c|c|}
\hline \multicolumn{2}{|c|}{ Treatment $^{\mathrm{z}}$} & \multicolumn{7}{|c|}{ Cumulative drop ( $\%$ of total on tree) } \\
\hline Chemical a.i. & $\mathrm{L} \cdot \mathrm{ha}^{-1}$ & 7 Sept. & 14 Sept. & 21 Sept. & 28 Sept. & 5 Oct. & 12 Oct. & 19 Oct. \\
\hline Control & 0 & $1 \mathrm{a}$ & $3 \mathrm{a}$ & $7 \mathrm{~b}$ & $15 \mathrm{~b}$ & $39 \mathrm{~b}$ & $82 \mathrm{ab}$ & $95 \mathrm{a}$ \\
\hline AVG $175 \mathrm{~g} \cdot \mathrm{ha}^{-1}$ & $189(2.5 \times)$ & $1 \mathrm{a}$ & $1 \mathrm{~b}$ & $3 \mathrm{~b}$ & $8 \mathrm{~b}$ & $19 \mathrm{c}$ & $69 \mathrm{bc}$ & $91 \mathrm{ab}$ \\
\hline AVG $175 \mathrm{~g} \cdot \mathrm{ha}^{-1}$ & $284(1.67 \times)$ & $0 \mathrm{a}$ & $1 \mathrm{~b}$ & 1.b & $3 \mathrm{~b}$ & $11 \mathrm{c}$ & $47 \mathrm{e}$ & $83 \mathrm{~b}$ \\
\hline AVG $175 \mathrm{~g} \cdot \mathrm{ha}^{-1}$ & $379(1.25 \times)$ & $1 \mathrm{a}$ & $1 \mathrm{~b}$ & $2 b$ & $7 \mathrm{~b}$ & $23 \mathrm{c}$ & $66 \mathrm{~cd}$ & $89 \mathrm{ab}$ \\
\hline AVG $175 \mathrm{~g} \cdot \mathrm{ha}^{-1}$ & $473(1 \times)$ & $1 \mathrm{a}$ & $2 \mathrm{~b}$ & $4 b$ & $6 \mathrm{~b}$ & $13 \mathrm{c}$ & 52 de & $82 \mathrm{~b}$ \\
\hline NAA $10 \mathrm{mg} \cdot \mathrm{L}^{-1}$ & $473(1 \times)$ & $1 \mathrm{a}$ & 2.ab & $13 \mathrm{a}$ & $33 \mathrm{a}$ & $61 \mathrm{a}$ & $90 \mathrm{a}$ & $98 \mathrm{a}$ \\
\hline \multicolumn{9}{|l|}{ Significance } \\
\hline AVG & & NS & NS & $* *$ & $* * *$ & $* * *$ & $* * *$ & $* *$ \\
\hline AVG spray volume & & NS & NS & NS & $\mathrm{C}^{*}$ & $\mathrm{C}^{*}$ & $\mathrm{C}^{*}$ & NS \\
\hline AVG vs. control & & NS & $*$ & $*$ & NS & $* * *$ & $* * *$ & $*$ \\
\hline AVG vs. NAA & & NS & NS & $* * *$ & $* * *$ & $* * *$ & $* * *$ & $* *$ \\
\hline Control vs. NAA & & NS & NS & $*$ & $* *$ & $*$ & NS & NS \\
\hline
\end{tabular}

${ }^{\mathrm{z}} \mathrm{AVG}$ was applied 25 Aug. 1992 and naphthaleneacetic acid on 9 Sept. 1992.

ns, ${ }^{*}, * * * * *$ Nonsignificant or significant at $P=0.05,0.01$, or 0.001 , respectively; $\mathrm{C}=$ cubic response. 
Table 6. Effect of AVG formulation, time of application, and NAA on preharvest drop of 'McIntosh' apples, Maine, 1993, Expt. 4.

\begin{tabular}{|c|c|c|c|c|c|c|c|c|c|c|c|}
\hline \multirow{2}{*}{$\begin{array}{l}\text { Treatment } \\
\text { (g.ha-1 a.i.) }\end{array}$} & \multirow{2}{*}{$\begin{array}{c}\text { Application }{ }^{z} \text { time } \\
\text { (weeks before } \\
\text { harvest) }\end{array}$} & \multicolumn{10}{|c|}{ Cumulative drop (\%) } \\
\hline & & 20 Sept. & 22 Sept. & 24 Sept. & Sept. 27 & 29 Sept. & 1 Oct. & 4 Oct. & 6 Oct. & 8 Oct. & 10 Oct. \\
\hline Control & & $3 \mathrm{a}$ & $3.8 \mathrm{a}$ & $6 \mathrm{a}$ & $14 \mathrm{a}$ & $29 \mathrm{a}$ & $46 \mathrm{a}$ & $71 \mathrm{a}$ & $78 \mathrm{a}$ & $87 \mathrm{a}$ & $95 \mathrm{a}$ \\
\hline ABG 3097124 & $6,4,2$ & $0 \mathrm{~b}$ & $0 \mathrm{~b}$ & $1 \mathrm{bc}$ & $3 \mathrm{bc}$ & $5 \mathrm{~cd}$ & $8 \mathrm{~cd}$ & $17 \mathrm{~cd}$ & $21 \mathrm{~d}$ & $34 \mathrm{c}$ & $43 \mathrm{c}$ \\
\hline ABG 3097185 & 4,2 & $0 \mathrm{~b}$ & $0 \mathrm{~b}$ & $1 \mathrm{c}$ & $2 \mathrm{bc}$ & $3 d$ & $4 d$ & $8 \mathrm{~d}$ & 9 de & $13 \mathrm{~d}$ & $21 \mathrm{de}$ \\
\hline ABG 3097372 & 4 & $0 \mathrm{~b}$ & $0 \mathrm{~b}$ & $0 \mathrm{c}$ & $0 \mathrm{c}$ & $1 \mathrm{~d}$ & $1 \mathrm{~d}$ & $2 d$ & $6 \mathrm{de}$ & $9 \mathrm{~d}$ & $15 \mathrm{de}$ \\
\hline ABG 3168124 & $6,4,2$ & $0 \mathrm{~b}$ & $0 \mathrm{~b}$ & $1 \mathrm{c}$ & $1 \mathrm{c}$ & $3 \mathrm{~d}$ & $5 \mathrm{~d}$ & $9 d$ & $13 \mathrm{de}$ & $16 \mathrm{~d}$ & $24 \mathrm{~d}$ \\
\hline ABG 3168185 & 4,2 & $0 \mathrm{~b}$ & $0 \mathrm{~b}$ & $1 \mathrm{c}$ & $2 \mathrm{bc}$ & $3 \mathrm{~d}$ & $4 d$ & $5 \mathrm{~d}$ & $5 \mathrm{e}$ & $5 d$ & 8 de \\
\hline ABG 3168372 & 4 & $0 \mathrm{~b}$ & $0 \mathrm{~b}$ & $0 \mathrm{c}$ & $2 \mathrm{bc}$ & $2 \mathrm{~d}$ & $2 \mathrm{~d}$ & $3 \mathrm{~d}$ & $3 \mathrm{e}$ & $3 \mathrm{~d}$ & $4 \mathrm{e}$ \\
\hline ABG 3168124 & 4 & $2 a b$ & $3 a b$ & $4 \mathrm{ab}$ & $7 \mathrm{~b}$ & $12 \mathrm{bc}$ & $16 \mathrm{bc}$ & $29 \mathrm{c}$ & $38 \mathrm{c}$ & $54 \mathrm{~b}$ & $65 \mathrm{~b}$ \\
\hline NAA-10 $\mathrm{mg} \cdot \mathrm{L}^{-1}$ & 2,1 & $2 a b$ & $2 a b$ & $3 \mathrm{bc}$ & $4 \mathrm{~b}$ & $15 \mathrm{~b}$ & $20 \mathrm{~b}$ & $42 \mathrm{~b}$ & $54 \mathrm{~b}$ & $64 \mathrm{~b}$ & $78 \mathrm{~b}$ \\
\hline \multicolumn{12}{|l|}{ Significance } \\
\hline \multicolumn{2}{|c|}{ ABG 3097} & $* *$ & $* *$ & $* * *$ & $* * *$ & $* * *$ & $* * *$ & $* * *$ & $* * *$ & $* * *$ & $* * *$ \\
\hline \multicolumn{2}{|c|}{ ABG 3097 No. of applications } & NS & NS & NS & NS & NS & NS & $\mathrm{L}^{*}$ & $\mathrm{~L}^{* *}$ & $\mathrm{~L}^{* * *}$ & $\mathrm{~L}^{* * *}$ \\
\hline \multicolumn{2}{|c|}{ ABG 3168} & $* *$ & $* *$ & $* * *$ & $* * *$ & $* * *$ & $* * *$ & $* * *$ & $* * *$ & $* * *$ & $* * *$ \\
\hline \multicolumn{2}{|c|}{ ABG 3168 No. of applications } & NS & NS & NS & NS & NS & NS & NS & NS & NS & $\mathrm{L}^{*}$ \\
\hline \multicolumn{2}{|c|}{ ABG 3097 vs. ABG 3168} & NS & NS & NS & NS & NS & NS & NS & NS & $* *$ & $* *$ \\
\hline
\end{tabular}

${ }^{\mathrm{z}} \mathrm{AVG}$ was applied on 9 and 23 Aug. and 9, 23, and 6 Sept. and NAA on 6 and 13 Sept. 1993.

ns,,$* * * * * * *$ Nonsignificant or significant at $P=0.05,0.01$, or 0.001 , respectively; $\mathrm{L}=$ linear response.

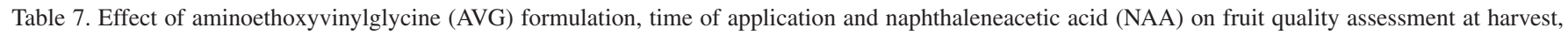
Maine, 1993.

\begin{tabular}{|c|c|c|c|c|c|c|c|c|c|c|}
\hline $\begin{array}{l}\text { Treatment }^{\mathrm{z}} \\
\left.\text { (g.ha }{ }^{-1} \text { a.i. }\right)\end{array}$ & $\begin{array}{c}\text { Application time } \\
\text { (weeks before } \\
\text { harvest) }\end{array}$ & $\begin{array}{l}\text { Fruit } \\
\text { wt } \\
(\mathrm{g})\end{array}$ & $\begin{array}{l}\text { Red } \\
\text { color } \\
(\%)\end{array}$ & $\begin{array}{l}\text { Fruit } \\
\text { size } \\
(\mathrm{mm})\end{array}$ & $\begin{array}{l}\text { Color } \\
\mathrm{L}^{*}\end{array}$ & $\begin{array}{l}\text { Hue } \\
\text { angle }\end{array}$ & $\begin{array}{c}\text { Flesh } \\
\text { firmness } \\
(\mathrm{N})\end{array}$ & $\begin{array}{l}\text { Soluble } \\
\text { solids } \\
(\%)\end{array}$ & $\begin{array}{l}\text { Starch } \\
\text { rating }\end{array}$ & $\begin{array}{c}\mathrm{IEC} \\
\left(\mu \mathrm{L} \cdot \mathrm{L}^{-1}\right)\end{array}$ \\
\hline \multicolumn{11}{|c|}{ Mean of harvests on 20 and 27 Sept. and 4 and 11 Oct. } \\
\hline 1) Control & & $164 b^{y}$ & $87 \mathrm{a}$ & $75 \mathrm{~b}$ & $34.7 \mathrm{~b}$ & $32.6 \mathrm{a}$ & $61.8 \mathrm{bc}$ & $11.5 \mathrm{a}$ & $7.2 \mathrm{ab}$ & $86 \mathrm{a}$ \\
\hline 2) NAA-10 $\mathrm{mg} \cdot \mathrm{L}^{-1}$ & 2,1 & $166 \mathrm{ab}$ & $86 a b$ & $75 \mathrm{~b}$ & $35.3 \mathrm{~cd}$ & $33.0 \mathrm{a}$ & $58.8 \mathrm{c}$ & $11.3 \mathrm{a}$ & $7.5 \mathrm{a}$ & $100 \mathrm{a}$ \\
\hline 3) ABG 3097123.5 & $6,4,2$ & $169 a b$ & $84 \mathrm{abc}$ & $76 \mathrm{ab}$ & $35.2 \mathrm{ab}$ & $32.7 \mathrm{a}$ & $65.5 \mathrm{ab}$ & $11.4 \mathrm{a}$ & $6.7 \mathrm{~b}$ & $39 \mathrm{bc}$ \\
\hline 4) ABG 3097185.3 & 4,2 & $182 \mathrm{a}$ & $81 \mathrm{bc}$ & $78 \mathrm{a}$ & $35.2 \mathrm{ab}$ & $34.2 \mathrm{a}$ & $64.6 \mathrm{ab}$ & $11.5 \mathrm{a}$ & $7.0 \mathrm{ab}$ & $21 \mathrm{de}$ \\
\hline 5) ABG 3097371.5 & 4 & $167 \mathrm{ab}$ & $83 \mathrm{abc}$ & $75 \mathrm{~b}$ & $35.5 \mathrm{ab}$ & $33.9 \mathrm{a}$ & $68.7 \mathrm{a}$ & $11.4 \mathrm{a}$ & $6.6 \mathrm{~b}$ & 26 cde \\
\hline 6) $\mathrm{ABG} 3168123.5$ & $6,4,2$ & $172 \mathrm{ab}$ & $82 \mathrm{abc}$ & $76 \mathrm{ab}$ & $36.0 \mathrm{a}$ & $34.6 \mathrm{a}$ & $66.4 \mathrm{ab}$ & $11.3 \mathrm{a}$ & $6.7 \mathrm{~b}$ & $33 \mathrm{~cd}$ \\
\hline 7) ABG 3168185.3 & 4,2 & $170 \mathrm{ab}$ & $80 \mathrm{c}$ & $76 \mathrm{ab}$ & $35.7 \mathrm{ab}$ & $33.9 \mathrm{a}$ & $66.3 \mathrm{ab}$ & $11.6 \mathrm{a}$ & $6.8 \mathrm{~b}$ & $14 \mathrm{e}$ \\
\hline 8) ABG 3168371.5 & 4 & $166 \mathrm{ab}$ & $84 \mathrm{abc}$ & $75 \mathrm{~b}$ & $34.9 \mathrm{ab}$ & $32.7 \mathrm{a}$ & $67.9 \mathrm{a}$ & $11.4 \mathrm{a}$ & $6.6 \mathrm{~b}$ & $11 \mathrm{e}$ \\
\hline 9) ABG 3168123.5 & 4 & $163 \mathrm{~b}$ & $87 \mathrm{ab}$ & $75 \mathrm{~b}$ & $35.2 \mathrm{ab}$ & $32.9 \mathrm{a}$ & $65.7 \mathrm{ab}$ & $11.5 \mathrm{a}$ & $6.9 \mathrm{ab}$ & $52 \mathrm{~b}$ \\
\hline \multicolumn{11}{|l|}{ Significance } \\
\hline AVG & & NS & NS & NS & NS & NS & $* *$ & NS & $*$ & $* * *$ \\
\hline Times of application & & $* * *$ & $* * *$ & $* * *$ & $* * *$ & $* * *$ & $* * *$ & $* * *$ & $* * *$ & $* * *$ \\
\hline AVG $\times$ time & & NS & NS & $*$ & NS & NS & $* * *$ & NS & $*$ & $* * *$ \\
\hline ABG 3097 & & NS & $*$ & NS & NS & NS & $*$ & NS & NS & $* * *$ \\
\hline ABG 3097 applicatio & & $*$ & NS & $*$ & NS & NS & NS & NS & NS & NS \\
\hline ABG 3168 & & NS & NS & NS & NS & NS & $* *$ & NS & $*$ & $* * *$ \\
\hline ABG 3168 applicatio & & NS & NS & NS & NS & NS & NS & NS & NS & $* *$ \\
\hline ABG 3097 vs. ABG 3 & & NS & NS & NS & NS & NS & NS & NS & NS & $*$ \\
\hline
\end{tabular}

${ }^{\mathrm{z}} \mathrm{AVG}$ was applied on 9 and 23 Aug. and 6 Sept. and NAA on 6 and 13 Sept. 1993.

ss,*,**,***N Nonsignificant or significant at $P=0.05,0.01$, or 0.001 , respectively.

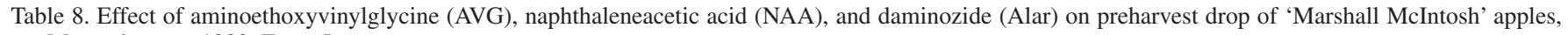
Massachusetts, 1993, Expt. 5.

\begin{tabular}{|c|c|c|c|c|c|c|c|c|}
\hline \multirow{2}{*}{$\begin{array}{l}\text { Treatment }^{\mathrm{z}} \\
\text { (g.ha }{ }^{-1} \text { a.i.) }\end{array}$} & \multirow{2}{*}{$\begin{array}{l}\text { Dates of } \\
\text { application }\end{array}$} & \multicolumn{7}{|c|}{ Cumulative drop $(\%)$} \\
\hline & & 13 Sept. & 20 Sept. & 27 Sept. & 4 Oct. & 11 Oct. & 18 Oct. & 25 Oct. \\
\hline Control & --- & $5 \mathrm{a}$ & $13 \mathrm{a}$ & $34 \mathrm{a}$ & $69 \mathrm{a}$ & $92 \mathrm{a}$ & $99 \mathrm{a}$ & $100 \mathrm{a}$ \\
\hline AVG 124 & 5 and 19 Aug., 2 Sept. & $1 \mathrm{~b}$ & $1 \mathrm{~b}$ & $4 \mathrm{c}$ & $8 \mathrm{c}$ & $36 \mathrm{~b}$ & $60 \mathrm{~b}$ & $82 \mathrm{~b}$ \\
\hline AVG 185 & 19 Aug., 2 Sept. & $1 \mathrm{~b}$ & $1 \mathrm{~b}$ & $3 \mathrm{c}$ & $7 \mathrm{c}$ & $22 \mathrm{~b}$ & $36 \mathrm{c}$ & $58 \mathrm{c}$ \\
\hline AVG 371 & 19 Aug. & $0 \mathrm{~b}$ & $2 \mathrm{~b}$ & $2 \mathrm{c}$ & $5 \mathrm{c}$ & $20 \mathrm{~b}$ & $31 \mathrm{c}$ & $53 \mathrm{c}$ \\
\hline AVG 124 & 19 Aug. & $1 \mathrm{~b}$ & $2 b$ & $13 \mathrm{bc}$ & $43 \mathrm{~b}$ & $80 \mathrm{a}$ & $91 \mathrm{a}$ & $96 \mathrm{a}$ \\
\hline NAA $10 \mathrm{mg} \cdot \mathrm{L}^{-1}$ & 9 Sept. & $3 a b$ & $5 \mathrm{~b}$ & $19 \mathrm{~b}$ & $60 \mathrm{a}$ & $91 \mathrm{a}$ & $97 \mathrm{a}$ & $99 \mathrm{a}$ \\
\hline Daminozide $750 \mathrm{mg} \cdot \mathrm{L}^{-1}$ & 5 Aug. & $1 \mathrm{~b}$ & $2 \mathrm{~b}$ & $5 \mathrm{bc}$ & $10 \mathrm{c}$ & $35 \mathrm{~b}$ & $55 \mathrm{~b}$ & $78 \mathrm{~b}$ \\
\hline \multicolumn{9}{|l|}{ Significance } \\
\hline AVG & & $* * *$ & $* * *$ & $* * *$ & $* * *$ & $* * *$ & $* * *$ & $* * *$ \\
\hline No. of AVG applications & & NS & NS & NS & NS & $* *$ & $* * *$ & $* * *$ \\
\hline AVG vs. NAA & & NS & NS & $*$ & $* * *$ & $* * *$ & $* * *$ & $* * *$ \\
\hline AVG vs. Daminozide & & NS & NS & NS & $* * *$ & $* * *$ & $* * *$ & $* * *$ \\
\hline
\end{tabular}

Ns,*,**,****Nonsignificant or significant at $P=0.05,0.01$, or 0.001 , respectively.

harvests was comparable regardless of formulation or number of applications. However, one application of AVG made 4 WBH more effectively controlled preharvest drop at the end of the season than an identical amount applied in split applications. AVG was superior to NAA in controlling drop after 27 Sept. AVG at $124 \mathrm{~g} \cdot \mathrm{ha}^{-1}$ a.i. applied three times controlled drop compara- bly to NAA, but it was less effective than when a total of $372 \mathrm{~g} \cdot \mathrm{ha}^{-1}$ a.i. was applied.

AVG slowed the loss of flesh firmness and reduced IEC (Table 7). However, AVG did not influence flesh firmness on the first harvest but on the last three evaluation dates AVG- treated fruit softened less than control fruit. IEC from all apples receiving $371.5 \mathrm{~g} \cdot \mathrm{ha}^{-1}$ a.i. was uni- formly low for the first three harvest dates (data not shown). However, IEC of fruit receiving one application at $4 \mathrm{WBH}$ was less than from those receiving similar amounts but in multiple applications. Although significant, the effect of AVGon starch degradation was small and erratic over time. AVG formulations were comparable except that IEC was less on ABG-3168 on the 
Table 9. Effect of aminoethoxyvinylglycine (AVG), naphthaleneacetic acid (NAA) and daminozide (Alar) on quality of 'Marshall McIntosh' apples at four harvests made between 16 Sept. and 7 Oct. 1993, Massachusetts.

\begin{tabular}{|c|c|c|c|c|c|c|}
\hline $\begin{array}{l}\text { Treatment } \\
\text { (g.ha-1 a.i.) }\end{array}$ & $\begin{array}{l}\text { Application } \\
\text { time } \\
1993\end{array}$ & $\begin{array}{l}\text { Fruit } \\
\text { wt } \\
(\mathrm{g})\end{array}$ & $\begin{array}{l}\text { Red } \\
\text { color } \\
(\%)\end{array}$ & $\begin{array}{l}\text { Flesh } \\
\text { firmness } \\
(\mathrm{N})\end{array}$ & $\begin{array}{l}\text { Soluble } \\
\text { solids } \\
(\%)\end{array}$ & $\begin{array}{l}\text { Starch } \\
\text { rating }\end{array}$ \\
\hline \multicolumn{7}{|c|}{ Mean of harvests on 16,23 , and 30 Sept. and 7 Oct. } \\
\hline 1) Control & --- & $175 b^{y}$ & $89 \mathrm{~b}$ & $65.1 \mathrm{c}$ & $13.1 \mathrm{ab}$ & $6.4 \mathrm{~b}$ \\
\hline 2) AVG 124 & 5 and 19 Aug., 2 Sept. & $180 \mathrm{ab}$ & $85 \mathrm{c}$ & $68.3 \mathrm{ab}$ & $13.2 \mathrm{a}$ & $5.7 \mathrm{c}$ \\
\hline 3) AVG 185 & 19 Aug., 2 Sept. & $182 \mathrm{ab}$ & $82 \mathrm{~d}$ & $67.4 \mathrm{ab}$ & $13.0 \mathrm{abc}$ & $5.9 \mathrm{c}$ \\
\hline 4) AVG 371 & 19 Aug. & $184 \mathrm{a}$ & $82 \mathrm{~d}$ & $67.9 \mathrm{ab}$ & $12.9 \mathrm{bcd}$ & $5.8 \mathrm{c}$ \\
\hline 5) $\mathrm{AVG} 124$ & 19 Aug. & $184 \mathrm{a}$ & $87 \mathrm{c}$ & $65.6 \mathrm{c}$ & $13.0 \mathrm{abc}$ & $6.2 \mathrm{~b}$ \\
\hline 6) NAA $10 \mathrm{mg} \cdot \mathrm{L}^{-1}$ & 9 Sept. & $184 \mathrm{a}$ & $90 \mathrm{~b}$ & $63.8 \mathrm{~d}$ & $12.9 \mathrm{~cd}$ & $6.8 \mathrm{a}$ \\
\hline 7) Daminozide $750 \mathrm{mg} \cdot \mathrm{L}^{-1}$ & 5 Aug. & $180 \mathrm{ab}$ & $92 \mathrm{a}$ & $69.3 \mathrm{a}$ & $12.8 \mathrm{~d}$ & $6.0 \mathrm{c}$ \\
\hline \multicolumn{7}{|l|}{ Significance } \\
\hline AVG & & NS & $* * *$ & $* * *$ & $* * *$ & $* * *$ \\
\hline Times of application & & $* * *$ & $* * *$ & $* * *$ & $* * *$ & $* * *$ \\
\hline $\mathrm{AVG} \times$ time & & NS & $* *$ & $* * *$ & NS & $*$ \\
\hline No. of applications & & NS & $* * *$ & NS & $*$ & NS \\
\hline AVG vs. NAA & & NS & $* * *$ & $* * *$ & NS & $* * *$ \\
\hline AVG vs. daminozide & & NS & $* * *$ & $* *$ & NS & NS \\
\hline
\end{tabular}

Ns,*,**,*** Nonsignificant or significant at $P=0.05,0.01$, or 0.001 , respectively.

last two harvest dates (data not shown).

Experiment 5. Concentration and multiple applications, Massachusetts. AVG retarded preharvest drop through the first two fruit evaluation periods to at least 27 Sept.(Table 8). Preharvest drop became severe after 4 Oct., but it was less on trees receiving one application of $371.5 \mathrm{~g} \cdot \mathrm{ha}^{-1}$ a.i. than on trees that received more than one application of lower rates of AVG. NAA and AVG at $123.5 \mathrm{~g} \cdot \mathrm{ha}^{-1}$ a.i. retarded drop minimally. AVG at $371.5 \mathrm{~g} \cdot \mathrm{ha}^{-1}$ a.i. and $750 \mathrm{mg} \cdot \mathrm{L}^{-1}$ daminozide retarded drop comparably until 27 Sept., but after that AVG was more effective.

AVG delayed the development of red color but there was a treatment $\times$ time interaction (Table 9). However, while red color was retarded by AVG by as much as $10 \%$ on the first two harvest dates, with additional time on the tree, the difference in color between AVG-treated and control fruit diminished to become small or nonsignificant (data not shown). AVG-treated fruit were firmer than control fruit, but there was an AVG $\times$ harvest time interaction. There was only a small difference in fruit flesh firmness at the early harvest dates, but later in the season AVG- treated fruit lost firmness less rapidly so that they were firmer at the later harvest dates (data not shown). AVG retarded the degradation of starch but there was an AVG $\times$ time of harvest interaction. With time on the tree, differences between AVG-treated and control fruit diminished (data not shown). Degradation of starch in AVG-treated fruit was retarded. Fruit treated with daminozide were firmer and had more red color than AVG-treated fruit. Fruit receiving NAA had more red color but were less firm and contained less starch than AVGtreated fruit.

\section{Discussion}

Bangerth (1978) first suggested that AVG may be a useful compound to control preharvest drop in apples. This study confirms that observation as well as the recent reports by Byers (1997a, 1997b) and Greene (2002a). Daminozide was the primary drop control compound used on 'McIntosh' because it not only controlled preharvest drop effectively but it also increased red color and flesh firmness at harvest (Edgerton et al., 1967; Southwick et al., 1968). Drop control by AVG was comparable to that caused by daminozide throughout the whole harvest period (Table 8). When drop was followed later into the season, AVG was more effective than daminozide. NAA remains registered as a drop control compound on apples. It has had a history of erratic response (Greene et al., 1987) that was confirmed in this investigation. In one experiment NAA accelerated drop (Table 5), in another one it had no effect (Table 4), while in two others it was marginally effective in retarding drop (Tables 6,8). Only in Expt. 1 (Table 1), did NAA produce commercially acceptable preharvest drop control, and in this experiment AVG was superior.

The results we obtained were without the use of an organosilicone surfactant, which have since been shown to increase the efficacy of AVG application, thereby allowing the use of lower concentrations. Greene (2002b) showed that 62 and $124 \mathrm{~g} \cdot \mathrm{ha}^{-1} \mathrm{AVG}$ was effective for delaying fruit maturity and preharvest drop of 'McIntosh' apples when applied with $0.1 \%$ (v/v) Silwet L-77 organosilicone surfactant.

Efficacy of most plant growth regulators is dependent upon good spray coverage. The most uniform spray coverage is often achieved when growth regulators are applied in a dilute spray $(1 \times)$. Since dilute applications are very time consuming, growers attempt to apply the appropriate dose in a smaller water volume to save time and money associated with application costs. While AVG applied in all spray volumes caused effective drop control, there was a linear decrease in efficacy with a reduction in spray volume (Table 1). Further, fruit from trees sprayed with a lower volume of water started to produce a substantial amount of ethylene earlier then fruit from trees where AVG was applied at $1 \times$ or $2 \times$ water volume. This can be interpreted to mean that spray coverage was not as good with the lower water volumes. Based upon these observations we suggest that a $2 \times$ spray volume is a good compromise between AVG efficacy and cost savings in application.

The easiest and most convenient way to apply
AVG is in one application. In this investigation one application made $4 \mathrm{WBH}$ was more effective at controlling preharvest drop than applying a comparable amount split before and after the 4 weeks before anticipated harvest date. Byers (1997a) reported that a split application made 4 and 2 WBH on 'Delicious' had better drop control than one application made $4 \mathrm{WBH}$. In another study using 'Golden Delicious' and 'Law Rome', Byers (1997b) reported that AVG application 4 weeks or 4 plus 2 WBH resulted in comparable drop control.

Preharvest application of AVG can reduce ethylene production and retard ripening in a number of apple cultivars (Autio and Bramlage, 1982; Bangerth, 1978; Bramlage et al., 1980, Schupp and Greene, 2004). The mode of action of AVG is through inhibition of ethylene biosynthesis (Boller et al., 1979) and delayed ripening is believed due to reduced ethylene production. The magnitude of response to AVG is cultivar dependent, with 'McIntosh' being one of the least responsive cultivars because it is a high ethylene-producing cultivar (Autio, and Bramlage, 1982; Bramlage et al., 1980). Delay in ripening can manifest itself by a reduction in red color, retardation in the loss of flesh firmness, and a delay in starch hydrolysis. In this investigation AVG did not uniformly and consistently delay ripening. Red color was reduced in 3 of 5 experiments (Expts. 4 and 4, Table 9), loss of flesh firmness was slowed in 4 of 5 experiments (Tables 2, 5, 7, and 9), and starch hydrolysis was reduced in 3 of 5 experiments (Tables 2, 7, and 9). The lack of a uniform response on ripening was also reported by Byers (1997a, 1997b).

'McIntosh' must be harvested over 2 to 2.5 weeks for optimum quality and storage potential. 'McIntosh' is also the dominant cultivar grown in New York and New England so a large volume of fruit must be harvested in a short period of time. A delay in ripening of selected blocks will be important in the timely and efficient harvest of quality apples. The challenge is to find optimum concentrations, times of application and identify weather conditions that will allow a consistent and uniform delay in ripening of this challenging cultivar. Fruit size at harvest may be influenced by the drop control compound used. Daminozide reduced fruit size, primarily as a carryover effect (Lord, 1971). NAA does not influence fruit size, but because it advances ripening, the harvest of high quality fruit usually must occur within 10 to $14 \mathrm{~d}$ of application (Southwick et al, 1953), thus limiting the time on the tree that the fruit has to size. AVG does not increase fruit size $\mathrm{per}$ se but larger fruit may be harvested on AVGtreated trees because harvest will be delayed longer than on nontreated trees. In all experiments reported in this investigation there was a highly significant effect of harvest date on fruit size. Fruit increase in size $\approx 1 \%$ per day that they remain on the tree near harvest (Batjer et al., 1957). Therefore, in most instances delaying harvest 2 to 3 weeks resulted in an increase in fruit size of $15 \%$ to $20 \%$. The increase in size may have an additional benefit if the increase in size moves fruit from one size category to a larger one that is selling at a higher price. 


\section{Literature Cited}

Autio, W.R. and W.J.Bramlage. 1982. Effects of AVG on maturation, ripening and storage of apples. J. Amer. Soc. Hort. Sci. 107:1074-1077.

Bangerth, F. 1978. The effect of a substituted amino acid on ethylene biosynthesis, respiration, ripening, and preharvest drop of apple fruits. J. Amer. Soc. Hort. Sci. 103:401-404.

Batjer, L. ., H.D. Billingsley, M.N. Westwood, and B.L. Rogers. 1957. Predicting harvest size of apples at different times during the growing season. Proc. Amer. Soc. Hort. Sci. 70:46-67.

Boller, T., R.C. Herner, and H. Kende. 1979. Assay for and enzymatic formation of an ethylene precursor, 1-aminocyclopropane-1-carborylic acid. Planta 145:293-303.

Bramlage, W.J., D.W. Greene, W.R. Autio, and J.M. McLaughlin. 1980. Effects of aminoethoxyvinylglycine on internal ethylene concentration and storage of apples. J. Amer. Soc. Hort. Sci. 105:847-851.

Byers, R.E. 1997a. Effects of aminoethoxyvinylglycine (AVG) on preharvest fruit drop and maturity of 'Delicious' apples. J. Tree Fruit Prod. 2:53-75.
Byers, R.E. 1997b. Effects of aminoethoxyvinylglycine (AVG) on preharvest fruit drop, maturity, and cracking of several apple cultivars. J. Tree Fruit Prod. 2:77-97.

Edgerton, L.P., C.G. Forshey, and G.D. Blanpied. 1967. Effect of summer application of Alar (B 995 ) in reducing drop and improving color and firmness of apples. Proc. N.Y. State Hort. Soc. 112:204-206.

Greene, D. W. 2002a. Preharvest drop control and maturity of 'Delicious' apples as affected by aminoethoxyvinylglycine (AVG). J. Tree Fruit Prod. 3(1):1-10.

Greene, D. W. 2002b. Preharvest drop control of 'McIntosh' apples with aminoethoxyvinylglycine and naphthaleneacetic acid. Quarterly Plant Growth Reg Soc. Amer. 30:121-130.

Greene, D.W., K. Kaminsky, and J. Sincuk. 1987. An evaluation of stop drop materials in 1986. Proc. Mass. Fruit Growers' Assoc. 93:74-78.

Lord, W.J. 1971. Effects of annual sprays of succinic acid-2,2-dimethlyhydiazide on vegetative growth, fruiting, fruit quality and preharvest drop of 'Delicious' apple trees. J. Amer. Soc. Hort. Sci. 96:687-690.
Marini, R.P., R.E. Byers, and D.L. Sowers. 1993. Repeat applications of NAA control preharvest drop of 'Delicious' apples. J. Hort. Sci. 68(2):247-253.

Priest, K.L. and E.C. Lougheed. 1981. Evaluating apple maturity using the starch-iodine test. Ont. Min. Agr. Food. Factsheet 81-025.

Schupp, J.R. and D.W. Greene. Effect of aminoethoxyvinylglycine(AVG) on preharvest drop, fruit quality, and maturation of 'McIntosh' apples: I. Effect of concentration and time of dilute applications. HortScience 39(5):1030-1035.

Shafer, W.E., R. Fritts, Jr., and B.N. Devisetty. 1995. A commercial formulation of AVG for use on apples. Proc. Wash. State Hort. Mtg. 91:169-171.

Southwick, F.W., I.E. Demoranville, and J.F. Anderson. 1953. The influence of some growth regulating substances on preharvest drop, color, and maturity of apples. Proc. Amer. Soc. Hort. Sci. 61:155-162.

Southwick, F.W., W.J. Lord, and W.D. Weeks. 1968. The influence of succinamic acid 2, 2-dimethylhydrazide (Alar) on the growth, productivity, mineral nutrition, and quality of apples. Proc. Amer. Soc. Hort. Sci. 92:71-81. 